\title{
Atenção integral à saúde de adolescentes em situação de trabalho: lições aprendidas
}

\author{
Com prehensive health services for working \\ adolescents: lessons learned
}

Carmen Ildes Rodrigues Fróes Asmus 1

Carm en Maria Raymundo 2

Suyanna Linhales Barker 2

Carla Cristina Coelho Augus to Pepe 2, 3

Maria Hel ena Ruzany 2

1 Núdeo de Estu dos de Saú de do Adolescen te, UERJ. Av. 28 de setembro 109, fundos, Vila Is a bel, 20551-030, Rio de Janei roRJ. nesa@uerj.br.

2 Núdeo de Estu dos da

Sa ú de do Adolescente, Uerj.

3 Núdeo de In formação

em Saúde do Trabalhador,

Fiocruz.
Abstract This article presents the collected experiences of the Health Program for Adolescent Workers (PSTA) at the Center for the Study of Adolescent Health at the State University of Rio de Janeiro. In terms of health services, the multidisciplinary team has devel oped a mod el of co mprehensive health care for adolescent workers that seeks to analyze the causal links between health problems and the wo rk conditions in which these young people are usually involved. In terms of health outreach, the health professionals have been wo rking with a group of adole scent wo rkers to develop a spe cific health edu ca tion methodology. This methodology seeks to promote a broad discussion of the meaning of wo rk in a empowering way that transcends the question of work. Based on this innovative experience, the staff was invited by the ILO to develop materials to train other professionals in the topics of health and occupational safety for adolescent workers. Given the current pol icies in effect in Brazil to eradicate child labor and protect adolescent workers, the experience of this initiative suggests the need for health programs to guarantee the rights of adolescent workers to occupational health and safety.

Key words Occupational health, Child labor, Health programs for adolescent workers
Resumo Este artigo apresenta a experiência do Programa de Saúde do Trabalhador Adolescen te (PSTA) do Núcleo de Estudos da Saúde do Adolescente da Uerj. Na área assistencial, a equipe multidisciplinar do Núcleo vem desenvolvendo um modelo de atenção integral aos adolescentes trabalhadores, buscando analisar as questões $l i-$ gadas ao nexo causal en tre os agravos à saúde e o processo produtivo em que estão envolvidos. No que se refere à exten são, osprofissionais, em associação com alguns adolescentes trabalhadores, vêm desenvolvendo uma metodologia de educação em saúde resgatando a discussão sob re o potencial produtivo de manei ra abrangente, abordando temas que vão além da questão do trabalho. Devido ao conhecimen to adquirido, a equipe, em parceria com a OIT, recebeu a incumbência de elaborar materiais pedagógi cos para a formação e capacitação de recursos humanos sobre saúde e segurança no trabalho infanto-juvenil. Conclui-seque, diantedas politicas públicas pa ra a eliminação do trabalho infantil e proteção do tra balhador adolescente, já em vigência em nivel nacional, a experiência relatada aponta pa ra a necessidade da criação de pro gramas semelhantes para a garantia dos direitos desta população.

Palavras-chave Saúde do trabalhador, Trabatho infanto-juvenil, Programa de saúde do tra balhador adolescente 


\section{Introdução}

A Organização Intern acional do Trabalho (OIT) estima que, no mun do, 352 milhões de crianças menores de 17 anos estejam trabalhando. Deste total, 8,4 milhões de crianças e adolescentes estão envolvidas com as piores formas de trabalho infantil, segundo definição da Convenção 182 desta instituição (ILO, 2002). A dimensão numérica, a importância da saúde física e psicossocial deste grupo populacional e as circunstâncias que aumentam as situações de risco a que se expõ em tornam a questão do trabalho infanto-juvenil uma prioridade na a genda das políticas públicas.

No Brasil, em função da ratificação das Convenções In ternacionais da OIT no 138 e no 182, respectivamente em 2001 e 2000 , o trabalho protegido de adolescentes na faixa etária en tre 16 e 18 anos é lícito. Para os que estão entre 14 e 16 anos, o trabalho só é admissível se eles estiverem na condição de aprendizes. Def ine-se como aprendiz o indivíduo com idade entre 14 e 16 anos que, por meio de bolsa de aprendizagem, faça parte de um programa de formação técnico-profissional, ministrado segundo as diretrizes e bases da legislação em vigor (arti go da lei 8.069/90). É con si derado trabalho infantil e, portanto, proibido por lei, qualquer trabalho realizado por indivíduos com menos de 14 anos.

A ex periência do trabalho precoce alimenta a idéia de que aqu ela atividade está ligada apenas à sobrevivência, em especial, pa ra as dasses populares. O mais preocupante é que, como em geral as seqüelas referentes às doenças do trabalho de adolescen tes aparecem somen te na fase adulta, as estatísticas ficam mascaradas, dificultando o desenvolvimen to de políticas de erradicação do trabalho infantil e pro teção do trabalho do adolescen te. Esta situação, de base socioeconômica e cultural, tem se refletido na saúde física e mental dos jovens, que cada vez mais se afastam da com preensão do significado social do trabalho e ficam potencialmente expos tos a ris cos oc u pacionais graves.

Contudo, muitos foram os avanços no comb a te ao probl ema do trabalho infantil, a respeito do qual as ex periências locais vêm-se articulando, completandoe potencializando Dentre esses avanços está o Programa Internacional para a Erradicação do Trabalho Infantil (IPEC) da OIT, que criou, em 2002, a Rede sobre o Trabalho Infantil Peri goso (Rede TIP), cujo obj etivo é fort a l ecer e amparar as ações destinadas à el i- minação do trabalho infantil nos países da Am é rica do Sul e Cen tral. No Brasil, destaca-se a el a boração de um plano naci onal para a erradicação do trabalho infantil pela Comissão Nacional para Erradicação do Trabalho Infantil (Con aeti), coorden ada pelo Ministério do Trabalho, em 2003.

Neste contexto, no início da década de 1990, o Núcl eo de Estudos da Sa ú dedo Adolescen te (Nesa) da Un ivers i d ade do Estado do Rio de Janei ro (Uerj) criou o Programa de Saúde do Trabalhador Adolescente (PSTA), que vem desenvolven do no campo da saúde pública uma metodologia de trabalho que articula saúde do adolescen te com a saúde do trabalhador. Este artigo tem por obj etivo relatar a ex periência e as lições aprendidas neste programa.

\section{Programa de Saúde do Trabalhador Adolescen te (PSTA)}

O PSTA/Nesa tem como obj etivo principal des envo lver estratégias de intervenção nas questões de saúde relacionadas ao processo de trabalho de crianças e adolescentes. A equipe do programa é multidisciplinar e composta por médico do trabalho, psicólogo, assistente social e sanitarista.

O programa tem os seguintes objetivos: prestar assistência integral aos adolescentes com agravos de saúde de origem ocupacional; de s envo lver metodo logias de iden tificação e investigação do processo saúde - doen ça - trabalho; realizar atividades de educação em saúde no senti do de criar espaços de deb a te sobre as situações de trabalho; produzir materiais educativos; formar e capacitar recursos humanos e des envo lver propostas de intervenção intersetorial e interinstitucional.

Os locais de atuação do PSTA/Nesa são: o Ambulatório de Saúde do Trabalhador Adolescente; programas que preparam adolescentes para o merc ado de trabalho; em presas que empregam adolescentes; cen tros comunitários de espaços popula res que de senvo lvem ativid ades de profissionalização com adolescentes.

O PSTA/Nesa participa, também, de ações interinstitu cionais com órgãos governa men tais e da socied ade civil, visando articular políticas preventivas e assistenciais voltadas à saúde do adolescen te trabalhador e pelo cumprimen to da legislação trabalhista vi gen te no país. 


\section{Ambulatório de atenção à saúde do adoles cen te trabalhador}

As ativid ades assistenciais do PSTA/Nesa ocorrem no Ambulatório de Saúde do Trabalhador Adolescen te situado na Policlínica Piquet Carneiro da Uerj. Além da equipe do programa, conta com o apoio técnico dos profissionais de saúde da Policlínica. Os adoles cen tes atendidos são geralmen te encaminhados por empresas, programas comunitários de inserção de jovens no mercado de trabalho, ou vêm por demanda espontânea. A maioria é oriunda do setor terciário (prestação de serviços) ou secundário (indústrias), exercen do ativid ades administra tivas.

En tre os anos de 1997 e 2002 foram aten didos 275 adolescentes, com idade média de 16 anos, sendo $93,5 \%$ (257) entre 14 e 17 anos e $6,5 \%$ (18) de 18 a 19 anos. A idade média de início da atividade laborativa foi de 14 anos (DP: 1.58). Na distribuição por sexo, 50,5\% (139) eram do sexo feminino e 49,5\% (136) do sexo masculino. A maiori a , 64,4\% (177) procurou nossos serviços por queixas de saúde gerais, seguido de $19,6 \%$ (54) encaminhados por projetos educativos de iniciação ao trabalho e $8,4 \%$ (23) por em presas.

Quando o adoles cente ch ega ao ambulatório é realizada uma anamnese ocupacional, e uma consulta clínica e social. Nesta ocasião, realizam-se grupos de reflexão e de informação sobre temas relativos ao processo de trabalho/saúde. Posteri ormente, caso seja necessário, o adoles cen te é encaminhado para especialistas médicos e outros profissionais de saúde, psicólogos, fonoaudiólogos, nutricionistas, fisio terapeutas.

$\mathrm{Na}$ identificação de um probl ema de saúde ocupacional, a equipe do programa procura um con ta to com o profissional responsável pelos adol e s cen tes no local de trabalho. Os con t atos estabelecidos são sem pre articulados com o Conselho Municipal da Criança e do Adolescente. Paralelamente, são desen cade adas as atividades de Vigilância Epidemiológica e Sanitária e de Inspeção do trabalho.

Os principais motivos de proc u ra ao ambulatório são questões gerais de saúde não originárias diretamen te do processo de trabalho. Embora, algumas vezes exista nexo causal en tre os problemas apresentados e a atividade laboral, comu m en te os adolescen tes não fazem esta vinculação. A anamnese ocupaci onal permi te $o$ estabelecim en to desta relação, bem como a detecção da exposição a agentes ambientais de ris- co à saúde. Da mesma forma, é um locus privilegiado de potencialização da percepção do adolescen te acerca das suas relações de trabalho, da forma como se vê como trabalhador, das suas ex pect a tivas qua n to a este trabalho e a sua vida profissional futura. Este processo favorece a discussão com o adolescen te sobre a complexidade das questões que envolvem o trabalho juvenil e ocasiona a discussão de con cei tos fundamentais em saúde do trabalhador, como aciden te de trabalho, situações de risco ocupacional, direitos do trabalhador adolescente.

A principal função exercida pelos adolescen tes aten di dosnesteambula tó rio é a de auxiliar administra tivo. As principais patologias relacionadas ao trabalho en contradas duran te o atendimen to de saúde são as doenças osteomusculares, em especial a lombalgia ocupacional, e a síndrome tensional do pescoço. Observou-se também uma gran de freqüência de infecções respiratórias de tra to su perior (sinusite, "gripe"), atopias (rinite, derm a ti te de contato), vícios (ou desvios) de refração (miopia, a stigma tismo) e hipertensão arterial.

As doenças osteomusculares estão associadas às condições do ambien te e às atividades desenvolvidas pelos adolescentes em funções administrativas, nas quais estão suj ei tos ao trabalho em ambien tes fechados, com refrigeração inadequada, e à falta e/ou dificuldade de aplicação das práticas ergonômicas à população adolescente trabalhadora. Entre os fatore s causais do processo de trabalho, pode-se citar: a execução de movimen tos repeti tivos exces sivamente rápidos, posturas viciosas e forçadas, deslocamen tos corporais forçados, con cen tração de força mecânica excessiva, e a inadequ ação dos instrumentos (ex: ferramentas), roupas eacessórios (Ministério da Saúde, 2001).

\section{Metodologias de educação em saúde}

Uma das estratégias desenvo lvidas pela equipe técnica do PSTA/Nesa é a criação de uma metodologia de educação em saúde (de promoção de saúde) para adolescen tes que resgate suas potencialidades com relação aos processos de produção ao qual estão inseridos.

Assim sendo, está sendo possível desenhar algumas formas de inserção destes jovens trabalhadores no processo de educação e saúde des envolvido pelo programa. Duas experiências de produção de metodologia e de material educativo com jovens trabalhadores do meio 
urbano são relatadas para exemplificar esta proposta metodológica.

Com o intuito de con h ecer melh or a população atendida, foi construído um grupo com adoles centes trabalhadores, para deb a ter temas rel acion ados à saúde. Em seguida, su geriu-se a inclusão de temas a serem discutidos com os jovens: "O que é ser adolescente"; "O Significado do trabalho"; "Aciden te do trabalho"; "Riscos ocupacionais"; "Saúde oral e sexualidade". A partir desta seleção de temas elaborou-se uma série de dinâmicas de gru po que facilitasse a reflexão de cada item. A regra básica deste processo é a de que nen hum assunto é discutido e/ou nen huma informação é dada antes que os adolescentes formulem perguntas sobre o tema e/ou rela tem alguma ex periência que ilu stre a questão a ser debatida. Técnicas de dramatização, de trabalho em pequenos grupos, audiovi suais e outras foram utilizadas para fomentar a participação dos jovens e a criação de um ambiente favorá vel para o surgimen to das dúvidas e suas re s postas - pelos técnicos ou pelos próprios joven s.

Como ilustração cita-se a dinâmica de grupo el a borada para discutir o tema "O significado do trabalho". A inclusão deste assun to surgiu da análise dos depoim en tos sobre os motivos que levavam os adoles cen tes a ingressar no mercado de trabalho. Por meio dos relatos, foi possível obs ervar que a família era a principal responsável pela inserção de s tes no merc ado de trabalho. Por esta razão, os adolescen tes não conseguiam esclarecer o que significava para eles mesmos o fato de começar a trabalhar. Eles descreviam esta situação como mais uma das obri gações que teriam que realizar para ajudar na econ omiadoméstica.

A partir desta constatação desenvolveu-se uma atividade de grupo na qual os jovens tinham de ex pressar com apenas uma palavra os motivos que os levaram a trabalhar. A dificuld ade de expressar, com uma só palavra, necessidades que não foram sentidas originalmente por eles, e sim pela família, fez com que o grupo começasse a discutir as razões que levaram $s$ eus familiares a inseri-los no mercado de trabalho.

Em seguida, os jovens foram incen tivados a elaborar uma série de idéias sobre que profissão gostariam de ter se pudessem escolher e o que poderiam fazer en quan to estavam naquele em prego, para alcançar o projeto desejado. $\mathrm{O}$ objetivo principal foi incluí-los como sujeitos na situação de trabalho, não reforçan do a con- cepção de que são apenas obj etos utilizados para suprir as necessidades das famílias. A proposta foi desenvolver coletivam en te estratégias de enfrentamento desta nova etapa de vida, a través da colocação das aspirações dos joven s som ada com a idéia de que profissão e trabalho podem ser atividades produtivas e prazerosas.

No de senvo lvimento desta atividade foi obs ervada a necessidade de produção de um material educativo de fácil assimilação pelos joven s, que con tivesse informações e dicas capazes de ajudar estes trabalhadores a enfrentarem certas dificuldades daqu ele processo de trabalho específico. Assim, foi produzida uma cartilha com uma linguagem própria para a faixa etária, para ampla distribuição. A confecção desta cartilha teve como premissa básica a participação dos adolescentes no seu processo de criação.

O trabalho consistiu em atividades que fizess em com que os adolescentes exercitassem s eus po tenciais criativos e a capacidade de trabalhar em equipe. A imaginação e a curiosidade também foram aproveitadas como elementos principais dos joven s. As técnicas utilizadas foram basicamen te atividades em que os jovens entraram em contato com suas capacidades de percepção corporal e cognitiva, e, a partir delas, foram criados novos conteúdos de expressão.

Os temas "Sa ú de do trabalhador" e "Adolescência" foram introduzidos para que fossem criadas histórias sobre estes conteúdos. Nesta fase, o processo ficou muito tenso e repleto de discussões e desentendimentos, afinal os adolescen tes estavam recriando suas pró prias histórias e isso, por vezes, fazia com que o limite entre a realidade e a fantasia ficasse imperceptível para alguns.

Todo o conteúdo da cartilha foi criado pelos jovens, desde o ti po físico dos personagen s até o desfecho da história.

\section{Educação permanen te em saúde}

O PSTA/Nesa articula ações interinstitucionais com outros setores da socied ade. A equ i pe procura desenvolver um processo de educação continuada para profissionais de saúde, obj etivando a sensibilização e capacitação progressiva na detecção dos agravos à saúde de ori gem ocupacional. Propicia também a participação de graduandos e pós-graduandos da Un ivers i d ade do Estado do Rio de Janei ro nas ativid ades ambulatoriais e nas dinâmicas de grupo realizadas. 
Além disso, o PSTA/Nesa real izaSeminários de Cap acitação Profissional sobre Sa ú deIn tegral do Adolescen te Trabalhador, dirigidos para profissionais de diversas insti tuições das áreas afins. Nestes even tos são abord ados temas referen tes não só à identificação dos ris cos ocupacionais do ambiente de trabalho e às principais patologias que acometem os adole s cen tes trabalhadores, mas também os aspectos de sexualidade, saúde oral e de crescimen to e de senvo lvimen to físico e emocional da fase da adolescência. Com financiamento da Capes, foi realizado um programa de sen sibilização e capacitação em saúde do adolescen te trabalhador para os profissionais da rede básica de assistência do município do Rio de Ja n ei ro(Proj eto Iden tid ade).

Atenden do ao pedido da Rede TIP da OIT e do Ministério da Saúde, o programa produziu um módulo de auto-aprendizagem sobre saúde e segurança no trabalho infantil e juvenil. Trata-se de um material técnico - pedagógico dirigido a equipes multidisciplinares que a tuam na área de saúde do trabalhador. Neste material busca-se introduzir os aspectos conceituais e procedimentos básicos de saúde para a atenção, in tervenção e vigilância a esse grupo populacional em suas atividades de trabalho. $\mathrm{O}$ propósito é ajudar na ampliação dos conhecimen tos e na busca de soluções para os problemas concretos de saúde de crianças e adolescen tes em situação de trabalho. Nosso desafio foi criar um material que, em vez de esgotar assuntos, estimulasse a capacidade de observação, reflexão e crítica, e o plu ralismo de idéias.

\section{Discussão}

A partir destas experiências de trabalho com adolescen tes trabalhadores, a equipe do PSTA/ Nesa vem constatando que certas situações são perpetuadas em nossa sociedade. Entre elas, pode-se citar a falta de correlação entre o conteúdo programáti co e o universo cultural dos adole s cen tes de classes populares, criandoneste gru po a noção de que permanecer na escola não significa melhores chances de qualificação para o mercado de trabalho, nem melhoria da qualidade de vida. Além disso, a ex periência de trabalho representa a sensação de independência e constitui a base para a esfera de consumo - ainda que em patamares mínimos - de acesso aos bens valorizados socialmentee que a família não pode financiar. A esses aspectos somam-se as necessidades de sobrevivência e a crença de que o trabalho constitua elemen to disciplinador e preven tivo da marginalidade.

Em uma análise crítica do trabalho do PSTA/Nesa, é possível observar na prática alguns dos efei tos do trabalho precoce. Pôde-se acompanhar histórias de vida de jovens que, des de mu i to cedo, perdem a capacidade de sonhar. Jovens que ficam reduzidos ao cansaço do dia de trabalho, que não ac reditam em suas capacidades intelectuais ou que, por falta de estímulo, já não as usam com tantafreqüência. $\mathrm{O}$ que eles aprendem muito rápido é a lição de que para ganhar dinhei ro e sustento é necessária a sujeição às leis do mercado de trabalho, aos patrões, e a tu do que é con trol ado pelo capital, ou seja, pa ra qu em eles ven dem sua força de trabalho.

Crianças e adolescentes, também, podem ser levados ou forçados a realizar tarefas que são perigosas ou inseguras mesmo para os adultos. No entanto, freqüentemente é imposto a estes realizar trabalhos que são geralmente considerados segu ros para os adultos, mas que podem não o ser para crianças e adolescentes. Os dados apontam que adolescentes e crianças têm ris comaior do que os adultos de de s envo lver doenças ocupacionais, tan to de forma mais precoce quan to com maior gravidade (WHO, 1987). É fundamental ter o conhecimen to de que os adolescen tes se en contram em período de crescimento e desenvolvimento, com capacidade e limitações para o trabalho e com re ações orgânicas às substâncias tóxicas diferen te $s$ das dos adultos (Asmus, 1996). Há períodos críti cos no estágio de des envolvim en to de uma determinada estrutura orgânica ou funcional, no qual ela é mais sensível à lesão. O dano pode não ser evidente até um estágio bastante posterior da vida (ATSDR, 2001).

A exaustão corporal provocada por uma carga de trabalho além do suportável pelo organismo do indivíduo - fadiga ocupacional, muscular, visual - assoc i ada a um aporte nutricional insuficien te parecem ser os fatores precipitantes para o de senvo lvimento das patologias detect adas no ambulatório (As mus, 2001). O rápido crescimen to durante a adolescência impõe necessidades nutricionais muito grandes, especialmen te durante um e meio a dois anos, qua ndo o ri tmo de crescimen to ch ega ao máximo. Neste período as necessidades nutricionais podem chegar ao dobro do necessário du ran teo res to da adolescência (WHO, 1995).

Estes dados apontam para a necessidade de o profissional de saúde ter conhecimen to dos 
fa tores de ris co do ambien te de trabalho, a fim de inves tigar a possibilidade de exposição ocupacional durante o atendimen to de saúde de crianças e adolescentes. $\mathrm{O}$ trabalho diminui o tempo disponível do adolescen te para seu lazer, vida em família, educação, e para a oportunidade de estabelecer relações de convivência com seus pares e outras pessoas da comunidade em geral. Além disso, ex perimentam um papel conflit a n teno local de trabalho, e na comunidade, pois como trabalhadores, adolescentes e crianças são forçados a agir como adultos e, no entanto, não podem escapar de sua condição de sujei to em de s envo lvimento. Estes fatores são uma fon te de desgaste emocional, que pode afetar o de senvo lvim en to cogn i tivo e físico. Este sofrimento é fruto do esforço que os mais jovens fazem de não dei $x$ a rem seu mu n do lúdico e cria tivo ser usurpado pela re alid adedo trabalho. Vale ressaltar que o lúdico na infância está associado à imaginação, à criatividade, à i nven tivid ade e ao mun do fantasmáti co essencial para a busca de soluções para os diversos desafios ineren tes ao de s envo lvimento humano (Meire, 2000).

O papel do setor saúde na erradicação do trabalho infantil e proteção do trabalhador adole s cen te não estava definido de forma dara e objetiva, com iniciativas pontuais surgindo externamen te ao governo (Nobre, 2003). As políticas de saúde do adolescen te estavam voltadas para as doenças infecciosas e para a violência; e as de saúde do trabalhador para as intoxicações químicas e para os aciden tes de trabalho. A partir de 2002, essas duas áreas vêm de s envo lven do políticas que visam à identificação e erradicação do trabalho infantil. A iniciativa do PSTA/Nesa/Uerj vem ao encontro desses esforços, propondo um modelo de atenção capaz de dar conta da complexidade da problem á tica apontada.

Seg un do Minayo Gomez (1989), uma das altern a tivas de tra n sormação para este quad ro é a possibilidade de criação de pedagogias educacionais e conte ú dos questionadores da atual organização do trabalho, propiciando assim que trabalh adores experimentem a realização de tarefas que não sejam apenas baseadas em disciplina, monotonia, desqualificação, com petição etc. Ne s te sen ti do é de grande importância para a integridade física e mental dos jovens traba$1 \mathrm{~h}$ adores que eles tenham acesso às informações sobre saúde do trabalhador, bem como sobre s eus direi tos à qualidade de trabalho. Estas informações devem ser transmitidas por meio de m etodo logias participativas, nas quais o processo de aprendizagem colabore para a formação de uma consciência crítica nos jovens.

\section{Considerações finais}

A experiência adquirida ao longo dos anos de trabalho no PSTA/Nesa permite à equipe determinar alguns pres su po stos básicos: crianças e adolescentes men ores de 14 anos não devem exercer nen huma ativid ade laboral, por causa dos importantes riscos que as cargas físicas, psíquicas e sociais do trabalho acarretam para o desenvolvimen to das suas potencialidades culturais, em ocionais e biológicas.

En tre os ado le s cen tesmaiores de 14 anos, é fundamental uma supervisão próxima das tarefas desempenhadas, de forma não só a prevenir po tenciais ri s cos à saúde, como também garantir uma formação técnico-profissional, e, assim, a construção de uma massa crítica ao seu papel como trabalhador.

Partindo destas premissas, o PSTA/Nesa tem buscado altern a tivas para o enfrentamento da questão adolescente - saúde - trabalho por m eio da implantação das atividades descritas. No entanto, compreende que, para um maior e s copo de atuação, algumas linhas de ação são prioritárias, de forma a possibilitar maior fiscalização do cumprimen to da legislação de proteção ao trabalho do menor, visando melhor con tribuir para a completa eliminação do trabalho de crianças e adolescen tes sob condições inadequadas.

(1) Sensibilização e capacitação de profess ores da rede municipal e estadual de educação para a abordagem da questão saúde/trabalho dentro das escolas, com o desenvo lvimen to de metodologias educativas apropriadas à cada realidade social. É importante a criação de espaços de discussão com os profe s sores, pois estes ocupam posição-chave na formação e transmissão do conhecimen to e das normas culturais da soci ed ade. São el ementos fundamen tais, portanto, para atuar na promoção da saúde do trabalhador, em sua fase mais inicial, propiciando um maior intercâmbio com a família e a comunidade e permitindo a con strução coletiva de alternativas viá veis que impeçam as conseqüências nocivas do trabalho precoce.

(2) Sen s ibilização e capacitação perm a n ente de profissionais da rede municipal de saúde para a identificação dos riscos do ambien te de trabalho, permitindo a detecção precoce dos 
a gravos à saúde de origem ocupacional e a sua con seqüente notificação. A inexistência de padronização na coleta de dados referen tes à incidência dos agravos à saúde de origem ocupacional, especificamente na população entre 10 e 17 anos, impede que se verifique a freqüência de morbimortalidade nesta população para identificar possíveis relações com os riscos do processo de trabalho. A subnotificação rem ete ao fato de que os profissionais de saúde, em sua maioria, não estão prep a rados para atender adequ adamente à população adolescente trabalhadora, que, muitas vezes, procura a unidade de saúde para tratar problemas que são ocasionados pelo seu ambiente de trabalho. A dificuldade de identificação do nexo etiológico, a imprecisa caracterização diagnóstica, a falta de escla recimen to do médico sobre a obriga toriedade de notificação da doença, a deficiência na formação de recursos humanos e a precariedade dos sis temas de Vigilância Epidemiológica exi s tentes são os fatores determinantes desta situação. Assim, torna-se urgen te a implem entação e efetivação desta rede de vigilância em saúde.

(3) O crescimen to da articulação com os cen tros comunitários das comu nidades e or ganizações não-governamentais por meio do a profundamen to do intercâ mbio com os projetos de formação profissional, em uma parceria comunidade/ $u$ n ivers idade/governo na con s trução coletiva de um modelo de atenção integral ao adolescen te trabalhador. Tal proposta permitiria que o conhecimen to desenvo lvi do pela academia fosse adequadamente oriundo da realidade da sociedade, contribuindo para o de senvo lvimento de altern a tivas metodológicas e para a aquisição de práticas realmen te eficazes na abordagem do trabalho de crianças e adolescen tes na nossa socied ade.

(4) A realização de pesquisas de inves ti gação do nexo causal en tre os agravos à saúde observados em subgrupos específicos de adolescentes trabalhadores, de forma a propor medidas de prevenção e con trole mais adequados à realid ade desta faixa et á ria.

É necessário um investimen to muito mais amplo para sanar o probl ema da ex pl oração de crianças e adolescen tes pelo trabalho. Entretanto, os serviços de saúde, as escolas e a sociedade em geral precisam estar atentos, dirigindo parte dos seus esforços para a resolução desta questão. $\mathrm{O}$ trabalho que o PSTA vem executando, tendo como base os pressupostos acima descritos, está direci on ado para o pon to de vi sta da saúde, com priorid ade para a con strução de conhecimentos sobre o tema, de modo a oferecer subsídios para a criação de políticas públicas. E, ainda, para a abertura de espaços on de os jovens possam elaborar sua participação na sociedade como trabalhadores. Desta forma, espera-se estar contribuindo para denunciar e modificar este quad ro.

\section{Colaboradores}

Todos os autores participaram igualmen te na ela boração do arti go, na discussão da proposta em equipe, na red ação e revisão do texto. 


\section{Referências bibliográficas}

As mus CF 2001. Aval i ação do processo produ tivo em mineração de diamantes e suas repercussões sobre a saúde dos adolescen tes garimpeiros. Tese de do utorado. Coordenação dos Programas de Pós-graduação em Engenharia, Universidade Federal do Rio de Janeiro, Rio de Janeiro.

Asmus CF, Ruzany MH, Barker SL \& Mei relles ZV 1996. Ri s cos oc u paci onaisna infância eadole scên cia. Uma revisão. J de Pediatria 72(4):203-208.

ATSDR (Agency for Toxic Substances and Disease Registry) 2001. Public Health Assessment Guidance [ Ma nual on line]. Disponível em $<$ http://www.atsdr. cdc.gov/HAC/HAGM/index.html $>$.

Barker SL \& Ru z a nyMH 1996. Metodologias participativas e jovens trabalhadores: o desafio de promover saúde ocupacional. Doc u m en to restrito. Rio de Janeiro, Nesa/ Uerj.

Hersztan J 1994. Considerations of susceptible populations, pp. 104-115. In L Rosenstock \& M Cullen (ed.) Textbook of Clinical Occupational and Environmental Medicine. WB Saunders, Philadelphia.

Intern a tional Labor Organization (ILO) 2002. Every child counts: new gl obal estimates on child labou r. In tern ational Labour Organization, Geneva.

Meire AMG 2000. Quando o trabalho da criança é o brincar, pp. 162-171. In A Jerusalinsky et al. O valor simbólico do trabalho e o su jei to contemporâneo. Artes e Ofícios, Porto Alegre.

Min ayo Gomez C 1989. Trabalhoe con hecimento: dilemas na educação do trabalhador. Cortez, São Paulo.

Ministério da Sa úde 2001. Doenças relacionadas ao trabalho: manual de procedimen tos para os serviços de saúde/Ministério daSa ú de do Brasil, Repre s entação no Brasil da Opas/OMS. Elizabeth Costa Dias (org.). Ministéro da Sa ú de do Brasil/Sec ret a ria de Políticas de Saúde/Departamento de Ações Progra má ticas Estratégicas/Área técnica de Sa ú de do Trabalhador. Bra sília DF.
Ministério do Trabalho 1978. Normas Regulamen tadoras de Segurança e Medicina do Trabalho. Norma Regulamentadora no 15 , anexo 3. Portaria 3.214 do Ministério do Trabalho (08/06/1978).

Mnistério do Trabalho 1996. Diagnóstico preliminar dos focos do tra balho da criança e do adolescen te no Brasil. Ministério do Trabalho, Brasília.

National Institute for Occupational Safety and Health/ Cen ter Disease Control 1996. Work-related injuries and illnesses associated with child labor - United States. JAMA 276(1):16-7.

Nobre LCC 2003. Trabalho de crianças e adole scentes: os desafios da intersetorialidade e o papel do Sistema Único de Saúde. Ciência \& Saúde Coletiva 8(4):963971.

Selevan SG, Kimmel CA \& Men dola P 2000. Identifying critical windows of exposu re for child ren's health. Environmental Health Perspective 108(su ppl 3):451455.

Sichieri R, Recine E \& Everhart JE 1995. Growth and body mass index of Brazilians ages 9 throwgh 17 years. Obes Res 3(Suppl. 2):117-121.

Tchernitchin AN \& Tch ernitchin N 1992. Imprinting of paths of h eterodiferentiati on by prenatal or neonatal exposu reto hormones, pharmaceuticals, pollutants and other agents and conditions. Medicine Science Research 20:391-397.

WHO Expert Committee on Physical Status 1995. The use and interpret ati on of Physical Status: the use and interpretation of Anth ropom etry. Technical Report Series 854:263-374.

WHO Study Group 1987. Children at Work: Special Health Risks. Technical Report Series 765:5-47.

Artigo apre s en tado em 18/10/2004

Aprovado em 16/03/2005

Versão final apre sen $\mathrm{t}$ ada em 18/04/2005 